ESAIM: PROCEEDINGS, March 2012, Vol. 35, p. 234-238

Fédération Denis Poisson (Orléans-Tours) et E. Trélat (UPMC), Editors

\title{
CERTIFIED METAMODELS FOR SENSITIVITY INDICES ESTIMATION
}

\author{
Alexandre Janon ${ }^{1}$, Mä̈lle Nodet ${ }^{1}$ And Clémentine Prieur ${ }^{1}$
}

\begin{abstract}
Global sensitivity analysis of a numerical code, more specifically estimation of Sobol indices associated with input variables, generally requires a large number of model runs. When those demand too much computation time, it is necessary to use a reduced model (metamodel) to perform sensitivity analysis, whose outputs are numerically close to the ones of the original model, while being much faster to run. In this case, estimated indices are subject to two kinds of errors: sampling error, caused by the computation of the integrals appearing in the definition of the Sobol indices by a Monte-Carlo method, and metamodel error, caused by the replacement of the original model by the metamodel. In cases where we have certified bounds for the metamodel error, we propose a method to quantify both types of error, and we compute confidence intervals for first-order Sobol indices.

Résumé. L'analyse de sensibilité globale d'un modèle numérique, plus précisément l'estimation des indices de Sobol associés aux variables d'entrée, nécessite généralement un nombre important d'exécutions $\mathrm{du}$ modèle à analyser. Lorsque celles-ci requièrent un temps de calcul important, il est judicieux d'effectuer l'analyse de sensibilité sur un modèle réduit (ou métamodèle), fournissant des sorties numériquement proches du modèle original mais pour un coût nettement inférieur. Les indices estimés sont alors entâchés de deux sortes d'erreur: l'erreur d'échantillonnage, causée par l'estimation des intégrales définissant les indices de Sobol par une méthode de Monte-Carlo, et l'erreur de métamodèle, liée au remplacement du modèle original par le métamodèle. Lorsque nous disposons de bornes d'erreurs certifiées pour le métamodèle, nous proposons une méthode pour quantifier les deux types d'erreurs et fournir des intervalles de confiance pour les indices de Sobol du premier ordre.
\end{abstract}

\section{Context}

\subsection{Monte-Carlo estimation of first-order Sobol indices}

Let $Y=f\left(X_{1}, \ldots, X_{p}\right)$ be our (scalar) output of interest, where the input variables $X_{1}, \ldots, X_{p}$ are modelised as independent random variables of known distribution. For $i=1, \ldots, p$, we recall the first-order Sobol index:

$$
S_{i}=\frac{\operatorname{Var}\left(\mathbf{E}\left(Y \mid X_{i}\right)\right)}{\operatorname{Var}(Y)}
$$

which measures, on a scale of 0 to 1 , the fraction of the total variability of the output caused by the variability in $X_{i}$ alone.

As $f$ is generally implicitly known ( $f$ can e.g. be a functional of a solution of a partial differential equation parametrized by functions of the input variables $X_{1}, \ldots, X_{p}$ ), one has no analytical expression for $S_{i}$ and has to resort to numerical estimation. The variances in the definition of $S_{i}$ can be expressed as multidimensional

\footnotetext{
${ }^{1}$ Laboratoire Jean Kuntzman, Université Joseph Fourier (Grenoble I), INRIA/MOISE, 51 rue des Mathématiques, BP 53, 38041 Grenoble cedex 9, France
}

(C) EDP Sciences, SMAI 2012 
integrals over the input parameters space. We use Monte-Carlo estimates for multidimensional integrals: let $\left\{\mathbf{X}^{k}\right\}_{k=1, \ldots, N}$ and $\left\{\mathbf{X}^{\prime k}\right\}_{k=1, \ldots, N}$ be two independent, identically distributed vector samples of $\mathbf{X}=\left(X_{1}, \ldots, X_{p}\right)$. For $k=1, \ldots, N$, we note:

$$
y_{k}=f\left(\mathbf{X}^{k}\right) \quad \text { and } \quad y_{k}^{\prime}=f\left(X_{1}^{\prime k}, \ldots, X_{i-1}^{\prime k}, X_{i}^{k}, X_{i+1}^{\prime k}, \ldots, X_{p}^{\prime k}\right) .
$$

We take the following statistical estimator for $S_{i}$, introduced in [3]:

$$
\widehat{S}_{i}(\mathcal{E})=\frac{\frac{1}{N} \sum_{k=1}^{N} y_{k} y_{k}^{\prime}-\left(\frac{1}{N} \sum_{k=1}^{N} y_{k}\right)\left(\frac{1}{N} \sum_{k=1}^{N} y_{k}^{\prime}\right)}{\frac{1}{N} \sum_{k=1}^{N}\left(y_{k}\right)^{2}-\left(\frac{1}{N} \sum_{k=1}^{N} y_{k}\right)^{2}}
$$

where $\mathcal{E}=\left(\left\{\mathbf{X}^{k}\right\}_{k=1, \ldots, N},\left\{\mathbf{X}^{\prime k}\right\}_{k=1, \ldots, N}\right)$ is our couple of samples the estimator depends on.

\subsection{Reduced basis metamodels}

In order to apply the reduced basis metamodelling, we further assume that the output $f(\mathbf{X})$ depends on a function $u(\mathbf{X})$ where, for every input $\mathbf{X}, u(\mathbf{X})$ satisfies a $\mathbf{X}$-dependent partial differential equation (PDE).

To make things clear, let us consider an example: we take $p=2$, so that $\mathbf{X}=\left(X_{1}, X_{2}\right)$, and take for $u\left(t, x ; X_{1}, X_{2}\right)$ the solution of the following $\left(X_{1}, X_{2}\right)$-dependent initial-boundary value problem (viscous Burgers' equation):

$$
\left\{\begin{array}{c}
\frac{\partial u}{\partial t}+\frac{1}{2} \frac{\partial}{\partial x}\left(u^{2}\right)-\nu \frac{\partial^{2} u}{\partial x^{2}}=1 \\
u(t=0, x)=u_{0 m}^{2}+5 \sin (0.5 x) \forall x \in[0 ; 1] \\
u(t, x=0)=b_{0} \\
u(t, x=1)=b_{1}
\end{array}\right.
$$

where our input parameters are $\left(X_{1}, X_{2}\right)=\left(\nu, u_{0 m}\right)$, and $b_{0}$ and $b_{1}$ are so that we have compatibility conditions: $b_{0}=u_{0 m}^{2}$ and $b_{1}=u_{0 m}^{2}+5 \sin (0.5)$.

This problem can be analyzed by means of the Cole-Hopf substitution (see [4] for instance), which turns the equation of interest into heat equation, leading to an integral representation of $u$ and well-posedness for $u \in C^{0}\left([0, T], H^{1}(] 0,1[)\right)$.

Note that the $x$ symbol denotes the spatial variable $u(\mathbf{X})$ depends on, and is unrelated with the parameters $X_{1}$ and $X_{2}$. Our output can be, for instance: $f(\mathbf{X})=\int_{0}^{T} \int_{0}^{1} u(t, x, \mathbf{X}) \mathrm{d} x \mathrm{~d} t$.

For a given value of $\mathbf{X}, u(\mathbf{X})$ is generally approximated using a numerical method, such as the finite-element method. These methods work by searching for $u(\mathbf{X})$ in a linear subspace of high dimension $\mathcal{N}$; this leads to a large linear system (or a succession of linear systems) to solve for the coefficients of (the approximation of) $u(\mathbf{X})$ in a fixed basis of $X$. This gives what we call the "full" discrete solution, that we denote again by $u(\mathbf{X})$. Even if efficient methods have been developed to solve the linear systems arising from such discretizations, the large number of unkowns that are to be found is often responsible for large computation times.

The reduced basis method is based on the fact that $\mathcal{N}$ has to be large because the basis we expand $u(\mathbf{X})$ in does not depend on the PDE problem that is being solved; hence it is too "generic": it can represent well a large number of functions, but allows much more degrees of freedom than wanted. We split the computation into two phases: the offline phase, where we seek a "reduced space", whose dimension $n$ is much smaller than $\mathcal{N}$, and which is suitable for effectively representing $u(\mathbf{X})$ for various values of the input parameter $\mathbf{X}$; and the online phase, where, for each required value of the input parameters, we solve the "projected" PDE on the reduced space.

This method is interesting if we are to solve the PDE for a number of values of the parameter sufficiently large so that the fixed cost of the offline phase is cancelled by the gain in marginal cost offered by the online phase vs. the standard discretization. This is often the case with Monte-Carlo estimations. 
One crucial feature of the reduced basis method, which we will rely on later, is that it provides a certified error bound $\epsilon_{u}(\mathbf{X}, t)$, which satisfies $\left(\|\cdot\|\right.$ being the usual norm on $\left.L^{2}([0,1])\right)$ :

$$
\|u(\mathbf{X} ; t)-\widetilde{u}(\mathbf{X} ; t)\| \leq \epsilon_{u}(\mathbf{X}, t) \quad \forall \mathbf{X}, \forall t \in[0 ; T]
$$

and, of course, $\epsilon_{u}$ can be fully and quickly computed (with a computation time of the same order of magnitude than the one for $\widetilde{u}$ ). This error bound on $u$ can lead to an error bound $\epsilon$ on the output:

$$
|f(\mathbf{X})-\widetilde{f}(\mathbf{X})| \leq \epsilon(\mathbf{X}) \quad \forall \mathbf{X}
$$

where $\widetilde{f}(\mathbf{X})$ denotes a functional of the reduced solution.

One can turn to [7] for a detailed introduction to the reduced basis method, and to [5] for the extension to the viscous Burgers equation (11).

\section{Construction of COMbined COnfidence intervals}

\subsection{Metamodel error}

For a couple of samples $\mathcal{E}=\left(\left\{\mathbf{X}^{k}\right\}_{k=1, \ldots, N},\left\{\mathbf{X}^{\prime k}\right\}_{k=1, \ldots, N}\right)$, we can use our metamodel output $\tilde{f}$ and our metamodel error bound $\epsilon$ to compute, for $k=1, \ldots, N$ :

$$
\widetilde{y}_{k}=\widetilde{f}\left(\mathbf{X}^{k}\right), \quad \widetilde{y}_{k}^{\prime}=\widetilde{f}\left(X_{1}^{\prime k}, \ldots, X_{i-1}^{\prime k}, X_{i}^{k}, X_{i+1}^{\prime k}, \ldots, X_{p}^{\prime k}\right)
$$

and:

$$
\epsilon_{k}=\epsilon\left(\mathbf{X}^{k}\right), \quad \epsilon_{k}^{\prime}=\epsilon\left(X_{1}^{\prime k}, \ldots, X_{i-1}^{\prime k}, X_{i}^{k}, X_{i+1}^{\prime k}, \ldots, X_{p}^{\prime k}\right)
$$

In [6], we show that we can compute rigorous and accurate bounds $\widehat{S}_{i}^{m}$ and $\widehat{S}_{i}^{M}$ depending only on the $\widetilde{y}_{k}, \widetilde{y}_{k}^{\prime}, \epsilon_{k}$ and $\epsilon_{k}^{\prime}$ so that:

$$
\widehat{S}_{i}^{m}(\mathcal{E}) \leq \widehat{S}_{i}(\mathcal{E}) \leq \widehat{S}_{i}^{M}(\mathcal{E})
$$

where $\widehat{S}_{i}(\mathcal{E})$ is the (unknown) value of the estimator of $S_{i}$ computed on the couple of samples $\mathcal{E}$. We emphasize that, in our approach, the $y_{k}$ and $y_{k}^{\prime}$ are not observed, as no evaluation of the full model is performed.

\subsection{Combined confidence intervals}

To take sampling error in account, we use a bootstrap procedure (see [1]) on the two bounding estimators $\widehat{S}_{i}^{m}$ and $\widehat{S}_{i}^{M}$. More specifically, we draw $N$ numbers with repetition from $\{1,2, \ldots, N\}$, so as to get a random list $L$. We then get two bootstrap replications by computing $\widehat{S}_{i}^{m}$ and $\widehat{S}_{i}^{M}$ using the samples couple $\left(\left\{\mathbf{X}^{k}\right\}_{k \in L},\left\{\mathbf{X}^{\prime k}\right\}_{k \in L}\right)$ instead of $\left(\left\{\mathbf{X}^{k}\right\}_{k=1, \ldots, N},\left\{\mathbf{X}^{\prime k}\right\}_{k=1, \ldots, N}\right)$. We repeat those computations for a fixed number $B$ of times, so as to obtain $B$ couples of replications $S_{i}^{m, 1}, \ldots, S_{i}^{m, B}$ and $S_{i}^{M, 1}, \ldots, S_{i}^{M, B}$. Now, for a fixed risk level $\left.\alpha \in\right] 0 ; 1[$, let $S_{i}^{\text {inf }}$ and $S_{i}^{\text {sup }}$ be, respectively, the $\alpha / 2$ quantile of $S_{i}^{m, 1}, \ldots, S_{i}^{m, B}$ and $S_{i}^{\text {sup }}$ be the $1-\alpha / 2$ quantile of $S_{i}^{M, 1}, \ldots, S_{i}^{M, B}$. We take $\left[S_{i}^{\text {inf }} ; S_{i}^{\text {sup }}\right]$ as our combined confidence interval for $S_{i}$. This confidence interval accounts for both metamodel and sampling error.

\subsection{Choice of sample size and reduced basis size}

Increasing $N$ and/or $n$ will increase the overall time for computation (because of a larger number of surrogate simulations to perform if $N$ is increased, or, if $n$ is increased, each surrogate simulation taking more time to complete due to a larger linear system to solve). However, increase in these parameters will also improve the precision of the calculation (thanks to reduction in sampling error for increased $N$, or reduction in metamodel error for increased $n$ ). In practice, one wants to estimate sensitivity indices with a given precision (ie. to produce $(1-\alpha)$-level confidence intervals with prescribed length), and has no a priori indication on how to 
choose $N$ and $n$ to do so. Moreover, for one given precision, there may be multiple choices of suitable couples $(N, n)$, balancing between sampling and metamodel error. We wish to choose the best, that is, the one who gives the smallest computation time.

On the one hand, we evaluate computation time: an analysis of the reduced basis method shows that the most costly operation made during a call to the metamodel is the resolution of a linear system of $n$ equations; this resolution can be done (e.g., by using Gauss' algorithm) with $O\left(n^{3}\right)$ operations. This has to be multiplied by the required number of online evaluations, i.e. the sample size $N$. Hence, we may assume that computation time is proportional to $N \times n^{3}$.

On the other hand, the mean length of the $(1-\alpha)$-level confidence intervals for $S_{1}, \ldots, S_{p}$ can be written as the sum of two terms. The first, depending on $N$, accounts for sampling error and can be modelled as $\frac{2 q_{\alpha} \sigma}{\sqrt{N}}$, where $\frac{\sigma}{\sqrt{N}}$ is the standard deviation of $\widehat{S}_{i}$ and $q_{\alpha}$ is an appropriate $\alpha$-dependent quantile of the standard gaussian distribution. The assumption of $1 / \sqrt{N}$ decay is heuristically deduced from central limit theorem.

The second term, which accounts for metamodel error, is assumed to be of exponential decay when $n$ increases: $C / a^{n}$, where $C>0$ and $a>1$ are constants. This assumption is backed up by numerical experiments as well as theoretical works [2].

We now wish to minimize computation time while keeping a fixed precision $p$ :

$$
\text { Find }\left(N^{*}, n^{*}\right)=\underset{(N, n) \in \mathbb{R}^{+} \times \mathbb{R}^{+}}{\operatorname{argmin}} n^{3} \times N \text { so that } \frac{2 q_{\alpha} \sigma}{\sqrt{N}}+\frac{C}{a^{n}}=p \text {. }
$$

The resolution of this problem is an elementary calculus argument. The solution involve the parameters $C$, $a$ and $\sigma$, which can be fitted against confidence interval lengths found during a "benchmark run".

\section{NumericAl RESUlts}

\subsection{Target model}

Our underlying model is given by the Burgers equation (1). Our output functional is taken to be: $f(\mathbf{X})=$ $\frac{1}{\mathcal{N}} \sum_{i=0}^{\mathcal{N}} u\left(t=T, x=\frac{i}{\mathcal{N}} ; \mathbf{X}\right)$.

We set $\mathcal{N}=60, \Delta t=.01, T=.05$, while the uncertain parameters $\nu$ and $u_{0 m}$ are assumed to be of uniform distributions, with respective ranges $[1 ; 20]$ and $[-0.3 ; 0.3]$. We also take $B=300$ bootstrap replications and a risk level $\alpha=0.05$.

Note that more flexible parametrizations of right-hand sides in (1) can be considered; results remain qualitatively the same. We chose this parametrization for simplicity reasons.

\subsection{Convergence benchmark}

Figure 1 shows the lower $\widehat{S^{m}}$ and upper $\widehat{S^{M}}$ bounds for different reduced basis sizes $n$ and fixed sample of size $N=300$, as well as the endpoints of the combined confidence intervals. This figure exhibits the fast convergence of our bounds to the true value of $S_{a}$ as the reduced basis size increases. We also see that the part of the error due to sampling (gaps between confidence interval upper bound and upper bound, and between confidence interval lower bound and lower bound) remains constant, as sample size stays the same.

\subsection{Comparison with estimation on the full model}

To demonstrate the interest of using sensitivity analysis on the reduced model, we computed the combined intervals for the two sensitivity indices using sample size $N=22000$ and $n=11$ (those parameters are found using the procedure described in Section 2.3 for a target precision $p=0.02$ ). We found [0.0674128; 0.0939712] for sensitivity index for $\nu$, and $[0.914772 ; 0.926563]$ for sensitivity with respect to $u_{0 m}$. These confidence intervals have mean length: $0.019 \approx 0.02$ as desired. This computation took $58.77 \mathrm{~s}$ of CPU time to complete (less than $1 \mathrm{~s}$ being spent in the offline phase). 


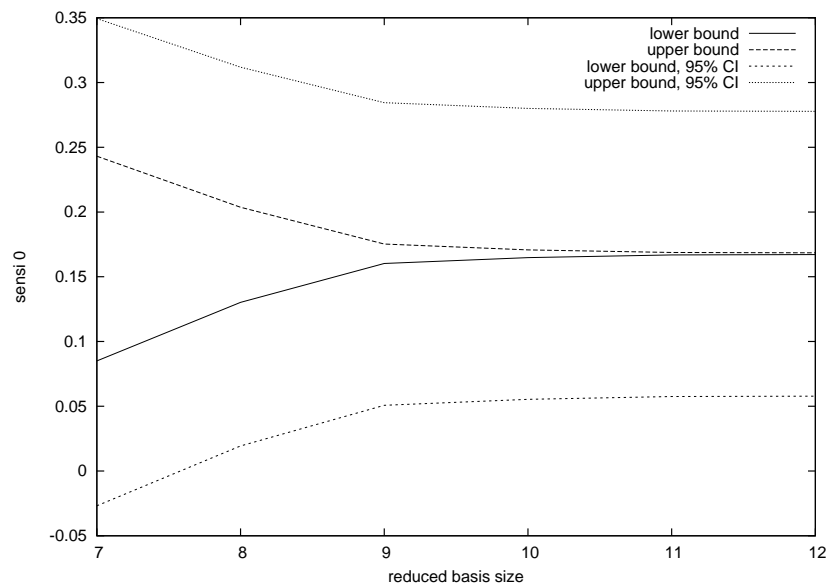

FiguRE 1. Convergence benchmark for sensitivity index of $\nu$. We plotted, for a fixed sample of size $N=300$, estimator bounds $\widehat{S}^{m}$ and $\widehat{S}^{M}$, and endpoints of the $95 \%$ combined confidence interval, for different reduced basis sizes.

To obtain a result of the same precision, we carry a simulation on the full model, for $N=22000$ (sample size can be chosen smaller than before, as there will be no metamodel error); we get a bootstrap confidence interval with mean length of $\approx 0.0193$ (we can only provide a confidence interval, as the exact values of the sensitivity indices are not known in this case). This computation takes $294 \mathrm{~s}$ of CPU time to complete. Hence, on this example, using a reduced-basis model roughly divides overall computation time by a factor of 5 , without any sacrifice on the precision and the rigorousness (as our metamodel error quantification procedure is fully proven and certified) of the confidence interval. We expect higher time savings with more complex (for example, twoor three-dimensional in space) models.

This work has been partially supported by the French National Research Agency (ANR) through COSINUS program (project COSTA-BRAVA no ANR-09-COSI-015).

\section{REFERENCES}

[1] GEB Archer, A. Saltelli, and IM Sobol. Sensitivity measures, ANOVA-like techniques and the use of bootstrap. Journal of Statistical Computation and Simulation, 58(2):99-120, 1997.

[2] A. Buffa, Y. Maday, A.T. Patera, C. Prud'homme, and Turinici G. A priori convergence of the greedy algorithm for the parametrized reduced basis. Mathematical Modelling and Numerical Analysis, 2009.

[3] T. Homma and A. Saltelli. Importance measures in global sensitivity analysis of nonlinear models. Reliability Engineering ES System Safety, 52(1):1-17, 1996.

[4] E. Hopf. The partial differential equation ut+ uux $=\mu x x$. Communications on Pure and Applied Mathematics, 3(3):201-230, 1950.

[5] A. Janon, M. Nodet, and C. Prieur. Certified reduced-basis solutions of viscous Burgers equations parametrized by initial and boundary values. Preprint available at http://hal.inria.fr/inria-00524727/en 2010, submitted.

[6] A. Janon, M. Nodet, and C. Prieur. Confidence intervals for sensitivity indices using reduced-basis metamodels. Preprint available at http://hal.inria.fr/inria-00567977/en 2011, submitted.

[7] N.C. Nguyen, K. Veroy, and A.T. Patera. Certified real-time solution of parametrized partial differential equations. Handbook of Materials Modeling, pages 1523-1558, 2005. 